\title{
A New Formulation of Maxwell's Equations in Clifford Algebra
}

\author{
Pirooz Mohazzabi ${ }^{1}$, Norbert J. Wielenberg1, Gary Clark Alexander ${ }^{2}$ \\ ${ }^{1}$ Department of Mathematics and Physics, University of Wisconsin-Parkside, Kenosha, WI, USA \\ ${ }^{2}$ Department of Mathematical Sciences, DePaul University, Chicago, IL, USA \\ Email: mohazzab@uwp.edu
}

How to cite this paper: Mohazzabi, P., Wielenberg, N.J. and Alexander, G.C. (2017) A New Formulation of Maxwell's Equations in Clifford Algebra. Journal of Applied Mathematics and Physics, 5, 1575-1588.

https://doi.org/10.4236/jamp.2017.58130

Received: July 27, 2017

Accepted: August 25, 2017

Published: August 28, 2017

Copyright () 2017 by authors and Scientific Research Publishing Inc. This work is licensed under the Creative Commons Attribution International License (CC BY 4.0).

http://creativecommons.org/licenses/by/4.0/

\begin{abstract}
A new unification of the Maxwell equations is given in the domain of Clifford algebras with $i_{j}^{2}=-1$ in a fashion similar to those obtained with Pauli and Dirac algebras. It is shown that the new electromagnetic field multivector can be obtained from a potential function that is closely related to the scalar and the vector potentials of classical electromagnetics. Additionally it is shown that the gauge transformations of the new multivector and its potential function and the Lagrangian density of the electromagnetic field are in agreement with the transformation rules of the second-rank antisymmetric electromagnetic field tensor, in contrast to the results obtained by applying other versions of Clifford algebras.
\end{abstract}

\section{Keywords}

Clifford Algebra, Maxwell's Equations, Electromagnetism, Vector, Potential

\section{Introduction}

Clifford algebras provide a unifying structure for Euclidean, Minkowski, and multivector spaces of all dimensions. Vectors and differential operators expressed in terms of Clifford algebras provide a natural language for physics which has some advantages over the standard techniques [1]-[6]. Applications of Clifford algebras and related spaces to mathematical physics are numerous. A valuable collection is given by Chishom and Common [4]. There are other applications in the literature. For example, DeFaria et al. [7] applied Clifford algebras to set up a formalism for magnetic monopoles. Salingaros [8] extended the Cauchy-Riemann equations of holomorphy to fields in higher-dimensional spaces in the framework of Clifford algebras and studied the Maxwell equations in vacuum 
and the Lorentz gauge conditions. He showed that the Maxwell equations in vacuum are equivalent to the equation of holomorphy in Minkowski space-time. Imaeda [9] showed that Maxwell equation in vacuum are equivalent to the condition of holomorphy for functions of a real biquaternion variable.

It has been shown that when the electromagnetic field is defined as the sum of an electric field vector and a magnetic field bivector, the four Maxwell equations reduce into a single equivalent equation in the domain of Pauli and Dirac algebras [3] [4]. In this work, we apply a different Clifford algebra to the Maxwell equations of electromagnetism, and we show how this formulation relates to the classical theory in a straightforward manner resulting in two main formulas; the first is a simplistic rendering of Maxwell's equations in a short formula

$$
\partial F-\partial_{0}\left(F+F^{*}\right)=-\rho+J
$$

The second is the reconstruction of the combined electric and magnetic fields by a single transformation of the four-potential

$$
F=u \partial-\partial \wedge u
$$

Our investigation differs in approach from those in Hestenes and ChisholmCommon in its simplicity and ability to use a single potential function to correctly derive Maxwell's equations in a vacuum.

In what follows, we first lay out the theory of the Clifford algebra employed in this work. We then discuss its applications to electromagnetism and obtain a new electromagnetic field multivector, which is closely related to the scalar and vector potentials of the classical electromagnetics. We show that the gauge transformations of the new multivector and its potential function and the Lagrangian density of the electromagnetic field are all in agreement with the transformation rules of the rank-2 antisymmetric electromagnetic field tensor. Finally, we give the matrix representation of the electromagnetic field multivector and its Lorentz transformation.

\section{Theory}

Consider the Clifford algebra $\mathscr{C}_{n}$ over the field of real numbers $\mathbb{R}$ generated by the elements $i_{1}, i_{2}, \cdots, i_{n}$ with relations

$$
\begin{cases}i_{j}^{2}=-1 & \text { for } j \geq 1 \\ i_{j} i_{k}=-i_{k} i_{j} & \text { for } j \neq k\end{cases}
$$

and no others [1] [10]. As a vecor space over $\mathbb{R}$, the algebra $\mathscr{C}_{n}$ has dimension $2^{n}$. A basis for $C_{n}$ consists of all products of the form $i_{k_{1}} i_{k_{2}} \cdots i_{k_{m}}$, with $k_{1}<k_{2}<\cdots<k_{m}$ and $m \leq n$. The empty product is identified with the scalar 1 . There are $2^{n}$ such products, and an arbitrary element $x$ of $\mathscr{C}_{n}$ (called a multivector) is a linear combination of these products. If we write $J$ for a multiindex $\left(k_{1}, \cdots, k_{m}\right)$ and $E_{J}=i_{k_{1}} i_{k_{2}} \cdots i_{k_{m}}$, then $x=\sum_{J} x_{J} E_{J}$, where $x_{J} \in \mathbb{R}$ for all $J$. For instance, an arbitrary element of $C_{2}$ can be written as

$$
x=x_{0}+x_{1} i_{1}+x_{2} i_{2}+x_{12} i_{1} i_{2}
$$




$$
\begin{aligned}
& \text { An important subspace of } \mathscr{C}_{n} \text { is } \\
& \qquad \mathbb{R}_{1}^{n}=\left\{x_{0}+\sum_{j=1}^{n} x_{j} i_{j} \mid x_{j} \in \mathbb{R}, i_{j} \text { are basis elements of } \mathscr{C}_{n}\right\}
\end{aligned}
$$

which is isomorphic to the generalized Minkowski space $\mathbb{R}_{1}^{n}$. Notice that this is a subspace of dimension $n+1$ rather than dimension $n$ or $2^{n}$.

A product $i_{k_{1}} i_{k_{2}} \cdots i_{k_{m}}$ where $k_{1}<k_{2}<\cdots<k_{m}$, or any expression equivalent to a scalar multiple of it is called an $m$-blade. Let $\langle x\rangle_{m}$ be the sum of the $m$ blades of $x$, called the $m$-vector part of $x$, then

$$
x=\sum_{m}\langle x\rangle_{m}
$$

If $x=\langle x\rangle_{m}$ for some positive integer $m$, then $x$ is said to be homogeneous of grade $m$.

The inner and outer products of blades are defined as follows [1]: The inner product of an $r$-blade $U_{r}$ and as $s$-blade $V_{s}$ is

$$
U_{r} \vee V_{s}=\left\langle U_{r} V_{s}\right\rangle_{|r-s|}
$$

The outer product of $U_{r}$ and $V_{s}$ is

$$
U_{r} \wedge V_{s}=\left\langle U_{r} V_{s}\right\rangle_{r+s}
$$

By linearity, these definitions extend to $x \vee y$ and $x \wedge y$, where $x$ and $y$ are multivectors.

Some examples of inner and outer products are:

$$
\begin{aligned}
& i \vee i=j \vee j=k \vee k=-1 \\
& i \wedge i=j \wedge j=k \wedge k=0 \\
& i \vee j=i \vee k=j \vee k=0 \\
& i \wedge j=i j \quad i \wedge k=i k \quad j \wedge k=j k \\
& i \vee i j=-j \quad i \vee j k=0 \quad i \wedge i j=0 \quad i \wedge j k=i j k
\end{aligned}
$$

There are three important involutions on $C_{n} \quad[10]$ :

1) inversion: $x \mapsto x^{\prime}$ defined by $i_{k}^{\prime}=-i_{k}$ for $k \geq 1$

2) reversion: $x \mapsto x^{*}$ defined by $\left(i_{k_{1}} \cdots i_{k_{m}}\right)^{*}=i_{k_{m}} \cdots i_{k_{1}}$

3) conjugation: $x \mapsto \bar{x}$ defined by $\bar{x}=\left(x^{*}\right)^{\prime}=\left(x^{\prime}\right)^{*}$

Then it follows that $(x y)^{\prime}=x^{\prime} y^{\prime},(x y)^{*}=y^{*} x^{*}$, and $\overline{(x y)}=\overline{y x}$ for all $x$ and $y$ in $C_{n}$.

\section{Derivatives}

Let $\nabla$ be the differential operator $\nabla=\sum_{k=1}^{n} i_{k} \partial_{k}$, where $\partial_{k}=\partial / \partial x_{k}$. Let $\partial$ be the differential operator $\partial=\partial_{0}+\nabla$. Let $D$ be a domain in $\mathbb{R}_{1}^{n}$, and suppose that $f: D \rightarrow \mathscr{C}_{n}$ has continuous derivatives of whatever order the context requires. Then $\partial f$ and $f \partial$ are the left and right derivatives of $f$, respectively. In terms of components, these derivatives are defined by

$$
\left(i_{k} \partial_{k}\right)\left(f_{j} i_{j}\right)=\frac{\partial f_{j}}{\partial x_{k}} i_{k} i_{j} \quad\left(f_{j} i_{j}\right)\left(i_{k} \partial_{k}\right)=\frac{\partial f_{j}}{\partial x_{k}} i_{j} i_{k}
$$


It is straightforward to show that the following identities hold:

$$
\begin{gathered}
\bar{\partial}=\partial_{0}-\nabla \\
\nabla \cdot \nabla=\sum_{k=1}^{n} \partial_{k}^{2} \\
\nabla^{2}=-\sum_{k=1}^{n} \partial_{k}^{2} \\
\bar{\partial} \partial=\partial \bar{\partial}=\partial_{0}^{2}+\nabla \cdot \nabla=\partial_{0}^{2}-\nabla^{2} \\
\partial \vee \partial=\partial_{0}^{2}-\nabla \cdot \nabla=\partial_{0}^{2}+\nabla^{2}
\end{gathered}
$$

The Clifford algebra $C_{2}$ maybe written as the algebra $\left\{x_{0}+x_{1} i+x_{2} j+x_{3} k: x_{m} \in R\right\}$ where $i j=k, j k=i, k i=j$. If we identify $\mathbb{R}^{3}$ with the subspace spanned by $\{i, j, k\}$, then $C_{2}$ is the usual skew-field of quaternions with

$$
x^{-1}=\frac{1}{|x|^{2}}\left(x_{0}-x_{1} i-x_{2} j-x_{3} k\right)
$$

The geometric product on $\mathbb{R}^{3}$ satisfies the relation $x y=-x \cdot y+x \times y$, where $x \times y$ is the usual cross-product. However, since additional relations exist among $i, j$, and $k$, inner and outer products are not defined here.

If $F$ is a vector field on $\mathbb{R}^{3}$, then it is straightforward to show that

$$
\nabla F=-\nabla \cdot F+\nabla \times F
$$

Theorem 1. Suppose $F$ is a vector field on $\mathbb{R}^{3}$. Then $\partial F=0$ if and only if $F=\nabla \phi$, where $\phi$ is a real-valued harmonic function of $\left(x_{1}, x_{2}, x_{3}\right)$.

Proof. A vector field $F$ equals $\nabla \phi$ for a real-valued function $\phi$ if and only if $\nabla \times F=0$. The function $\phi$ is harmonic if and only if $\nabla \cdot F=0$.

\section{Applications to Electromagnetism}

In Gaussian units, the differential form of the Maxwell equations for sources in vacuum are [11]

$$
\begin{gathered}
\nabla \cdot E=\rho \\
\nabla \cdot B=0 \\
\nabla \times E+\frac{1}{C} \partial_{t} B=0 \\
\nabla \times B-\frac{1}{c} \partial_{t} E=J
\end{gathered}
$$

where $E, B$, and $J$ are time-dependent vector fields in $\mathbb{R}^{3}$ and $\rho$ is a real-valued function. That is, each quantity is a function of $\left(x_{0}, x_{1}, x_{2}, x_{3}\right)$, where $x_{0}=c t$ and $t$ is time. Note that $\rho$ is the charge density multiplied by $4 \pi$ and $J$ is the current density multiplied by $4 \pi / c$.

We recast the Maxwell equations in the language of Clifford algebras by keeping the electric field as a vector, but replacing the magnetic field vector by the magnetic field bivector $M$, defined as [3] [12] 


$$
M=B_{1} j k+B_{2} k i+B_{3} i j
$$

The electromagnetic field multivector is then defined as $F=E+M$. It can be shown that

$$
\begin{gathered}
\nabla E=\nabla \vee E+\nabla \wedge E \\
\nabla M=\nabla \vee M+\nabla \wedge M \\
\nabla \vee E=-\partial_{1} E_{1}-\partial_{2} E_{2}-\partial_{3} E_{3}=-\nabla \cdot E \\
\nabla \wedge E=\left(\partial_{2} E_{3}-\partial_{3} E_{2}\right) j k+\left(\partial_{3} E_{1}-\partial_{1} E_{3}\right) k i+\left(\partial_{1} E_{2}-\partial_{2} E_{1}\right) i j \\
\nabla \vee M=\left(\partial_{2} B_{3}-\partial_{3} B_{2}\right) i+\left(\partial_{3} B_{1}-\partial_{1} B_{3}\right) j+\left(\partial_{1} B_{2}-\partial_{2} B_{1}\right) k=\nabla \times B \\
\nabla \wedge M=\left(\partial_{1} B_{1}+\partial_{2} B_{2}+\partial_{3} B_{3}\right) i j k=(\nabla \cdot B) i j k
\end{gathered}
$$

In terms of $E$ and $M$, the Maxwell equations for sources in vacuum may now be written as

$$
\begin{gathered}
\nabla \vee E=-\rho \quad(\text { a scalar equation }) \\
\nabla \vee M-\partial_{0} E=J \quad(\text { a vector equation }) \\
\nabla \wedge E+\partial_{0} M=0 \quad \text { (a bivector equation) } \\
\nabla \wedge M=0 \quad(\text { a trivector equation })
\end{gathered}
$$

Theorem 2. The Maxwell equations are equivalent to the single equation

$$
\partial F-\partial_{0}\left(F+F^{*}\right)=-\rho+J
$$

Proof. Since $F=E+M$,

$$
\begin{aligned}
\partial F & =\partial E+\partial M=\partial_{0} E+\nabla \vee E+\nabla \wedge E+\partial_{0} M+\nabla \vee M+\nabla \wedge M \\
& =2 \partial_{0} E+(\nabla \vee E)+\left(\nabla \vee M-\partial_{0} E\right)+\left(\nabla \wedge E+\partial_{0} M\right)+(\nabla \wedge M)
\end{aligned}
$$

Using the Maxwell equations, we obtain

$$
\partial F=2 \partial_{0} E-\rho+J
$$

But

$$
E=\frac{1}{2}[(E+M)+(E-M)]=\frac{1}{2}\left(F+F^{*}\right)
$$

Therefore, we obtain Equation (32). Conversely, assuming Equation (32), the Maxwell equations follow by setting the real parts, the vector parts, the bivector parts, and the trivector parts of each side equal. This completes the proof.

From classical electrodynamics [11], the fields $E$ and $B$ are derived from a scalar potential $\phi$ and a vector potential $A$ by

$$
\begin{gathered}
E=-\frac{1}{c} \partial_{t} A-\nabla \phi \\
B=\nabla \times A
\end{gathered}
$$

where $\phi$ and $A$ satisfy the wave equations

$$
\left(\frac{1}{c^{2}} \partial_{t}^{2}-\nabla \cdot \nabla\right) \phi=\rho
$$




$$
\left(\frac{1}{c^{2}} \partial_{t}^{2}-\nabla \cdot \nabla\right) A=J
$$

and the continuity equation

$$
\frac{1}{c} \partial_{t} \phi+\nabla \cdot A=0
$$

We can formulate this as follows: Let $u=-\phi-A$, and write

$$
u=u_{0}+u_{1} i+u_{2} j+u_{3} k
$$

where $u_{0}=-\phi$ and $V e(u)=-A$. Then

$$
\begin{gathered}
E=-\partial_{0} A-\nabla \phi=\partial_{0}[A e(u)]+\nabla u_{0} \\
M=\nabla \wedge A=-\nabla \wedge[A e(u)]
\end{gathered}
$$

Note that $\partial_{0}=(1 / c) \partial_{t}$.

The derivative of $u$ is

$$
\partial u=\left(\partial_{0} u_{0}-\partial_{1} u_{1}-\partial_{2} u_{2}-\partial_{3} u_{3}\right)+\partial_{0}[A e(u)]+\nabla u_{0}+\nabla \wedge[A e(u)]
$$

Using Equations (42) and (43) and noting that

$$
\partial \vee u=\partial_{0} u_{0}-\partial_{1} u_{1}-\partial_{2} u_{2}-\partial_{3} u_{3}
$$

we obtain

$$
\partial u=\partial \vee u+E-M=\partial \vee u+F^{*}
$$

Theorem 3. The electromagnetic field $F$ is obtained from the potential function $u$ by

$$
F=u \partial-\partial \vee u
$$

Proof. From Equation (46) we have $F^{*}=\partial u-\partial \vee u$. Therefore,

$$
F=(\partial u)^{*}-(\partial \vee u)^{*}=u^{*} \partial^{*}-\partial \vee u=u \partial-\partial \vee u
$$

Note that Equation (47) may also be written as $F=u \partial-2 \partial_{0} u_{0}$, since by the continuity eqation $\partial_{0} u_{0}=-\partial_{1} u_{1}-\partial_{2} u_{2}-\partial_{3} u_{3}$.

\section{Gauges}

\subsection{Lorentz Transformation of the Electromagnetic Field}

A Lorentz transformation is an isometry $P$ of the Minkowski space $\mathbb{R}_{1}^{n}$, such that $\operatorname{Re}(A x)>0$ whenever $\operatorname{Re}(x)>0$. In the special case where one inertial reference frame $\left(y_{0}, y_{1}, y_{2}, y_{3}\right)$ is moving relative to another frame $\left(x_{0}, x_{1}, x_{2}, x_{3}\right)$ with constant velocity $v$ in the $x_{1}$-direction, the Lorentz transformation relating them is represented by the matrix

$$
P=\left(\begin{array}{cccc}
a & -b & 0 & 0 \\
-b & a & 0 & 0 \\
0 & 0 & 1 & 0 \\
0 & 0 & 0 & 1
\end{array}\right)
$$


where

$$
a=\frac{1}{\sqrt{1-(v / c)^{2}}} \quad \text { and } \quad b=\frac{a v}{c}=\frac{v / c}{\sqrt{1-(v / c)^{2}}}
$$

In the general case, writing $y=P x$, we have

$$
\frac{\partial u}{\partial x_{s}}=\sum_{r=0}^{3} \frac{\partial u}{\partial y_{r}} \frac{\partial y_{r}}{\partial x_{s}}
$$

and

$$
P_{r s}=\frac{\partial y_{r}}{\partial x_{s}}
$$

Thus the operator $\partial_{x}$ transforms as $\partial_{x} u=\left(\partial_{y} A\right) u$, where $A$ acts on $\partial_{y}$ on the right in the usual way. Calculations show that associativity does not hold in the expression. To summarize, if $y=P x$, then $\partial_{y} u=\left(\partial_{x} P^{-1}\right) u$.

Suppose now that $u=u(x)$, where $u: \mathbb{R}_{1}^{3} \rightarrow \mathbb{R}_{1}^{3}$ is a potential function for $F$ so that $F=u(x) \partial_{x}-\partial_{x} \vee u(x)$. Then $\hat{u}=P u\left(P^{-1} y\right)$ is a potential function for the transformed electromagnetic field multivector $\hat{F}$. Therefore,

$$
\hat{F}=\hat{u}(y) \partial_{y}-\partial_{y} \vee \hat{u}(y)
$$

and $\partial_{y}=\partial_{x} P^{-1}$.

Theorem 4. Under the Lorentz transformation $y=P x$, the electromagnetic field multivector $F$ transforms into $\hat{F}$ according to

$$
\hat{F}=(P u)\left(\partial_{x} P^{-1}\right)-\left(\partial_{x} P^{-1} \vee P u\right)
$$

Again, associativity does not hold in this equation.

\subsection{Lorenz Gauge Invariance}

Before we get to the mathematics of this section, let us note the difference in Lorentz and Lorenz. These names, in fact, do belong to different scientists and thus we consider both types of gauge invariance here.

The common gauge invariant from classical electrodynamics is to consider

$$
\begin{aligned}
& \phi^{\prime}=\phi-\frac{\partial f}{\partial t} \\
& A^{\prime}=A+\nabla f
\end{aligned}
$$

In our formalism this leads us to

$$
u^{\prime}=u+\bar{\partial} f
$$

Examining this a little more fully, we know that the electric and magnetic fields do not change under Lorenz or Coulomb gauges and thus we obtain

$$
F=u \partial-\partial v u=u^{\prime} \partial-\partial \vee u^{\prime}
$$

Following through we see

$$
\begin{aligned}
u^{\prime} \partial-\partial v u^{\prime} & =(u+\bar{\partial} f) \partial+\partial \vee(u+\bar{\partial} f) \\
& =(u \partial-\partial \vee u)+(\bar{\partial} f) \partial-\partial \vee(\bar{\partial} f)=F+(\bar{\partial} f) \partial-\partial \vee(\bar{\partial} f)
\end{aligned}
$$


As the multivector field must remain unchanged we obtain the gauge invariant condition

$$
(\bar{\partial} f) \partial-\partial \vee(\bar{\partial} f)=0
$$

\section{The Lagrangian Density}

Recall that in classical electromagnetism the Lagrangian density in a vacuum is given by

$$
\mathcal{L}=-\frac{1}{4}\left(F_{\mu \nu} F^{\mu \nu}\right)
$$

By expanding this a bit, we find

$$
\mathcal{L}=\frac{1}{2}\left(E^{2}-B^{2}\right)
$$

In order to recreate this in the Clifford algebraic formulation we consider

$$
F=E+B \quad \text { and } \quad F^{*}=E-B
$$

Thus we might expect that the Lagrangian density becomes

$$
\mathcal{L}=-\frac{1}{2}\left(F \vee F^{*}\right)
$$

Examining this a little we see that

$$
F \vee F^{*}=(E+B) \vee(E-B)=E^{2}-B^{2}-E \vee B+B \vee E
$$

Since our inner product is commutative we have a cancellation of field product terms $E \cdot B$ and $B \cdot E$.

In higher dimensions, one may wish to restrict to the 0-blade so as to disallow higher dimensional cross terms. Thus we write

$$
\mathcal{L}=-\frac{1}{2}\left\langle F \vee F^{*}\right\rangle_{0}
$$

Now let's consider the situation outside a vacuum. We have

$$
\mathcal{L}=-\frac{1}{4}\left(F_{\mu v} F^{\mu v}\right)+j_{\mu} A^{\mu}
$$

Let us write

$$
j=\rho+J
$$

Then using our potential $u$ we have the Lagrangian density of the electromagnetic fields outside a vacuum,

$$
\mathcal{L}=-\frac{1}{2}\left(F \vee F^{*}\right)+j \vee u
$$

\section{Representation by Matrices}

Complex numbers can be represented by $2 \times 2$ matrices. Similarly, as we show in the Appendix, the Clifford algebra $C_{3}$ is represented by $8 \times 8$ matrices. The element $x=x_{0}+x_{1} i+x_{2} j+x_{3} k+x_{4} j k+x_{5} k i+x_{6} i j+x_{7} i j k$ is represented by the 
matrix

$$
\left(\begin{array}{cccccccc}
x_{0} & -x_{1} & -x_{2} & -x_{3} & -x_{4} & -x_{5} & -x_{6} & x_{7} \\
x_{1} & x_{0} & -x_{6} & x_{5} & -x_{7} & -x_{3} & x_{2} & -x_{4} \\
x_{2} & x_{6} & x_{0} & -x_{4} & x_{3} & -x_{7} & -x_{1} & -x_{5} \\
x_{3} & -x_{5} & x_{4} & x_{0} & -x_{2} & x_{1} & -x_{7} & -x_{6} \\
x_{4} & -x_{7} & -x_{3} & x_{2} & x_{0} & -x_{6} & x_{5} & -x_{1} \\
x_{5} & x_{3} & -x_{7} & -x_{1} & x_{6} & x_{0} & -x_{4} & -x_{2} \\
x_{6} & -x_{2} & x_{1} & -x_{7} & -x_{5} & x_{4} & x_{0} & -x_{3} \\
x_{7} & x_{4} & x_{5} & x_{6} & x_{1} & x_{2} & x_{3} & x_{0}
\end{array}\right)
$$

From this we can write an $8 \times 8$ natrix representation of the electromagnetic field multivector

$$
F=E_{1} i+E_{2} j+E_{3} k+B_{1} j k+B_{2} k i+B_{3} i j
$$

The $4 \times 4$ matrix in the upper left corner contains all the coordinates of $F$ and is the same as the matrix representation of the second-rank antisymmetric electromagnetic field tensor [11]. If we use this representation for $F$, that is,

$$
F=\left(\begin{array}{cccc}
0 & -E_{1} & -E_{2} & -E_{3} \\
E_{1} & 0 & -B_{3} & B_{2} \\
E_{2} & B_{3} & 0 & -B_{1} \\
E_{3} & -B_{2} & B_{1} & 0
\end{array}\right)
$$

then the Lorentz transformation of $F$ is given by [11]

$$
\hat{F}=P F P^{t}
$$

A quite lengthy calculation (see Appendix) shows that the two transformations given by Equations (54) and (73) are exactly identical.

\section{Concluding Remarks}

We have shown that in the framework of the Clifford algebra defined in Equation (3), the Maxwell equations in vacuum reduce to a single equation in a fashion similar to that in other types of Clifford algebras. The multivector $F$ is closely related to the second-rank antisymmetric electromagnetic field tensor [11], whose condition of holomorphy is also equivalent to the Maxwell equations in vacuum [8]. However, the multivector formalism may have some theoretical advantages over the tensor formalism.

Furthermore, we have shown that the electromagnetic field multivector can be derived from a potential function $u$, which is closely related to the scalar and the vector potentials of classical electromagnetics.

Finally, we have discussed the Lorentz transformation of the potential function $u$ and the multivector field $F$, and have shown that these transformations are in agreement with the transformation of the second-rank antisymmetric electromagnetic field tensor.

The formulation given by other investigators [3] [4] differs from the present work in that they have employed the Pauli algebra in which the square of each of 
the three unit elements is +1 rather than -1 , or the Dirac algebra in which one unit element has square +1 and three unit elements have square -1 . All these types of Clifford algebras have been extensively used.

By repeating our calculations with $i_{j}^{2}=+1$ instead of -1 in Equation (3), it can be shown that the Maxwell equations in vacuum reduce to $\partial F=\rho-J$, which is in agreement with the result given by Jancewicz [12]. Equation (47) then becomes $F=u \partial$, where $u$ is the potential function given by Equation (41). Equation (54) for the Lorentz transformation of $F$ then reduces to $\hat{F}=(P u)\left(\partial P^{-1}\right)$. Repeating the calculations of the Appendix, it turns out that this transformation is equivalent to $\hat{F}=P F P^{-1}$, where the matrix representation of $F$ is now given by

$$
F=\left(\begin{array}{cccc}
0 & E_{1} & E_{2} & E_{3} \\
E_{1} & 0 & B_{3} & -B_{2} \\
E_{2} & -B_{3} & 0 & B_{1} \\
E_{3} & B_{2} & -B_{1} & 0
\end{array}\right)
$$

Note that this matrix is not antisymmetric and the representation is not the same as that of the electromagnetic field tensor, and the transformation rule is also different. This is in contrast to the result obtained from applying a Clifford algebra with $i_{j}^{2}=-1$.

\section{References}

[1] Hestenes, D. and Sobczyk, G. (1984) Clifford Algebra to Geometric Calculus. Reidel, Dordrecht. https://doi.org/10.1007/978-94-009-6292-7

[2] Hestenes, D. (1986) New Foundations for Classical Mechanics. Reidel, Dordrecht. https://doi.org/10.1007/978-94-009-4802-0

[3] Hestenes, D. (1966) Space-Time Algebra. Gordon and Breach, New York.

[4] Chisholm, J.S.R. and Common, A.K., Eds. (1986) Clifford Algebras and Their Applications in Mathematical Physics. Reidel, Dordrecht.

https://doi.org/10.1007/978-94-009-4728-3

[5] Kyrala, A. (1967) Theoretical Physics. Saunders, Philadelphia.

[6] Snygg, J. (1986) Expediting the Spinning Top Problem with a Small Amount of Clifford Algebra. American Journal of Physics, 54, 708-712. https://doi.org/10.1119/1.14510

[7] DeFaria, R.M.A., Recami, E. and Rodrigues Jr., W.A. (1985) A Satisfactory Formalism for Magnetic Monopoles by Clifford Algebras. Physics Letters B, 173, 233-236. https://doi.org/10.1016/0370-2693(86)90508-3

[8] Salingaros, N. (1981) Electromagnetism and Holomorphic Properties of Spacetime. Journal of Mathematical Physics, 22, 1919-1925. https://doi.org/10.1063/1.525165

[9] Imaeda, K. (1976) A New Foundation of Classical Electromagnetism. Nuovo Cimento $B$, 32, 138-162. https://doi.org/10.1007/BF02726749

[10] Ahlfors, L.V. (1985) Mobius Transformations and Clifford Numbers in "Differential Geometry and Complex Analysis, H. E. Rauch Memorial Volume." Springer-Verlag, Berlin, 65-73.

[11] Jackson, J.D. (1999) Classical Electrodynamics. 3rd Edition. Wiley, Hoboken. 
[12] Jancewicz B. (1986) Remarks on Cliford Algebra in Classical Electromagnetism. In: Chisholm, J.S.R. and Common, A.K., Eds., Clifford Algebras and Their Applications in Mathematical Physics, Reidel, Dordrecht, 485-493. 


\section{Appendix}

Here we show that the two transformations given in Equations (54) and (73) are identical.

We have

$$
x=x_{0}+x_{1} i+x_{2} j+x_{3} k+x_{4} j k+x_{5} k i+x_{6} i j+x_{7} i j k
$$

and

$$
y=y_{0}+y_{1} i+y_{2} j+y_{3} k+y_{4} j k+y_{5} k i+y_{6} i j+y_{7} i j k
$$

Therefore,

$$
\begin{aligned}
x y= & \left(x_{0} y_{0}-x_{1} y_{1}-x_{2} y_{2}-x_{3} y_{3}-x_{4} y_{4}-x_{5} y_{5}-x_{6} y_{6}+x_{7} y_{7}\right) \\
& +\left(x_{0} y_{1}+x_{1} y_{0}+x_{2} y_{6}-x_{3} y_{5}-x_{4} y_{7}+x_{5} y_{3}-x_{6} y_{2}-x_{7} y_{4}\right) i \\
& +\left(x_{0} y_{2}-x_{1} y_{6}+x_{2} y_{0}+x_{3} y_{4}-x_{4} y_{3}-x_{5} y_{7}+x_{6} y_{1}-x_{7} y_{5}\right) j \\
& +\left(x_{0} y_{3}+x_{1} y_{5}-x_{2} y_{4}+x_{3} y_{0}+x_{4} y_{2}-x_{5} y_{1}-x_{6} y_{7}-x_{7} y_{6}\right) k \\
& +\left(x_{0} y_{4}-x_{1} y_{7}+x_{2} y_{3}-x_{3} y_{2}+x_{4} y_{0}+x_{5} y_{6}-x_{6} y_{5}-x_{7} y_{1}\right) j k \\
& +\left(x_{0} y_{5}-x_{1} y_{3}-x_{2} y_{7}+x_{3} y_{1}-x_{4} y_{6}+x_{5} y_{0}+x_{6} y_{4}-x_{7} y_{2}\right) k i \\
& +\left(x_{0} y_{6}+x_{1} y_{2}-x_{2} y_{1}-x_{3} y_{7}+x_{4} y_{5}-x_{5} y_{4}+x_{6} y_{0}-x_{7} y_{3}\right) i j \\
& +\left(x_{0} y_{7}+x_{1} y_{4}+x_{2} y_{5}+x_{3} y_{6}+x_{4} y_{1}+x_{5} y_{2}+x_{6} y_{3}+x_{7} y_{0}\right) i j k
\end{aligned}
$$

It follows that the matrix representation of $y$ is

$$
\left(\begin{array}{cccccccc}
y_{0} & -y_{1} & -y_{2} & -y_{3} & -y_{4} & -y_{5} & -y_{6} & y_{7} \\
y_{1} & y_{0} & -y_{6} & y_{5} & -y_{7} & -y_{3} & y_{2} & -y_{4} \\
y_{2} & y_{6} & y_{0} & -y_{4} & y_{3} & -y_{7} & -y_{1} & -y_{5} \\
y_{3} & -y_{5} & y_{4} & y_{0} & -y_{2} & y_{1} & -y_{7} & -y_{6} \\
y_{4} & -y_{7} & -y_{3} & y_{2} & y_{0} & -y_{6} & y_{5} & -y_{1} \\
y_{5} & y_{3} & -y_{7} & -y_{1} & y_{6} & y_{0} & -y_{4} & -y_{2} \\
y_{6} & -y_{2} & y_{1} & -y_{7} & -y_{5} & y_{4} & y_{0} & -y_{3} \\
y_{7} & y_{4} & y_{5} & y_{6} & y_{1} & y_{2} & y_{3} & y_{0}
\end{array}\right)
$$

We also have

$$
F=u \partial-\partial \vee u=E_{1} i+E_{2} j+E_{3} k+B_{1} j k+B_{2} k i+B_{2} i j
$$

So the matrix representation of $F$ is

$$
F=\left(\begin{array}{cccccccc}
0 & -E_{1} & -E_{2} & -E_{3} & -B_{1} & -B_{2} & -B_{3} & 0 \\
E_{1} & 0 & -B_{3} & B_{2} & 0 & -E_{3} & E_{2} & -B_{1} \\
E_{2} & B_{3} & 0 & -B_{1} & E_{3} & 0 & -E_{1} & -B_{2} \\
E_{3} & -B_{2} & B_{1} & 0 & -E_{2} & E_{1} & 0 & -B_{3} \\
B_{1} & 0 & -E_{3} & E_{2} & 0 & -B_{3} & B_{2} & -E_{1} \\
B_{2} & E_{3} & 0 & -E_{1} & B_{3} & 0 & -B_{1} & -E_{2} \\
B_{3} & -E_{2} & E_{1} & 0 & -B_{2} & B_{1} & 0 & -E_{3} \\
0 & B_{1} & B_{2} & B_{3} & E_{1} & E_{2} & E_{3} & 0
\end{array}\right)
$$

The general Lorentz transformation and its inverse are given by the following matrices: 


$$
\begin{aligned}
P & =\left(\begin{array}{llll}
P_{00} & P_{01} & P_{02} & P_{03} \\
P_{10} & P_{11} & P_{12} & P_{13} \\
P_{20} & P_{21} & P_{22} & P_{23} \\
P_{30} & P_{31} & P_{32} & P_{33}
\end{array}\right) \\
P^{-1} & =\left(\begin{array}{cccc}
P_{00} & -P_{10} & -P_{20} & -P_{30} \\
-P_{01} & P_{11} & P_{21} & P_{31} \\
-P_{02} & P_{12} & P_{22} & P_{32} \\
-P_{03} & P_{13} & P_{23} & P_{33}
\end{array}\right)
\end{aligned}
$$

From Equation (54) we have

$$
\hat{F}=(P u)\left(\partial P^{-1}\right)-\left(\partial P^{-1}\right) \vee(P u)
$$

where

$$
\begin{aligned}
P u= & \left(P_{00} u_{0}+P_{01} u_{1}+P_{02} u_{2}+P_{03} u_{3}\right) \\
& +\left(P_{10} u_{0}+P_{11} u_{1}+P_{12} u_{2}+P_{13} u_{3}\right) i \\
& +\left(P_{20} u_{0}+P_{21} u_{1}+P_{22} u_{2}+P_{23} u_{3}\right) j \\
& +\left(P_{30} u_{0}+P_{31} u_{1}+P_{32} u_{2}+P_{33} u_{3}\right) k
\end{aligned}
$$

and

$$
\begin{aligned}
\partial P^{-1}= & \left(P_{00} \partial_{0}-P_{01} \partial_{1}-P_{02} \partial_{2}-P_{03} \partial_{3}\right) \\
& -\left(P_{10} \partial_{0}-P_{11} \partial_{1}-P_{12} \partial_{2}-P_{13} \partial_{3}\right) i \\
& -\left(P_{20} \partial_{0}-P_{21} \partial_{1}-P_{22} \partial_{2}-P_{23} \partial_{3}\right) j \\
& -\left(P_{30} \partial_{0}-P_{31} \partial_{1}-P_{32} \partial_{2}-P_{33} \partial_{3}\right) k
\end{aligned}
$$

Let $P_{m}$ be the $m$-th row of $P$. Then

$$
\begin{gathered}
P u=\left(P_{0} \cdot u\right)+\left(P_{1} \cdot u\right) i+\left(P_{2} \cdot u\right) j+\left(P_{3} \cdot u\right) k \\
\partial P^{-1}=\left(\bar{P}_{0} \cdot \partial\right)-\left(\bar{P}_{1} \cdot \partial\right) i-\left(\bar{P}_{2} \cdot \partial\right) j-\left(\bar{P}_{0} \cdot \partial\right) k
\end{gathered}
$$

and

$$
\begin{aligned}
F= & {\left[\left(P_{1} \cdot u\right)\left(\bar{P}_{0} \cdot \partial\right)-\left(P_{0} \cdot u\right)\left(\bar{P}_{1} \cdot \partial\right)\right] i } \\
& +\left[\left(P_{2} \cdot u\right)\left(\bar{P}_{0} \cdot \partial\right)-\left(P_{0} \cdot u\right)\left(\bar{P}_{2} \cdot \partial\right)\right] j \\
& +\left[\left(P_{3} \cdot u\right)\left(\overline{P_{0}} \cdot \partial\right)-\left(P_{0} \cdot u\right)\left(\bar{P}_{3} \cdot \partial\right)\right] k \\
& +\left[\left(P_{3} \cdot u\right)\left(\bar{P}_{2} \cdot \partial\right)-\left(P_{2} \cdot u\right)\left(\bar{P}_{3} \cdot \partial\right)\right] j k \\
& +\left[\left(P_{1} \cdot u\right)\left(\bar{P}_{3} \cdot \partial\right)-\left(P_{3} \cdot u\right)\left(\bar{P}_{1} \cdot \partial\right)\right] k i \\
& +\left[\left(P_{2} \cdot u\right)\left(\bar{P}_{1} \cdot \partial\right)-\left(P_{1} \cdot u\right)\left(\bar{P}_{2} \cdot \partial\right)\right] i j
\end{aligned}
$$

Recall that from Equation (42) we have

$$
E=\left(\partial_{0} u_{1}+\partial_{1} u_{0}\right) i+\left(\partial_{0} u_{2}+\partial_{2} u_{0}\right) j+\left(\partial_{0} u_{3}+\partial_{3} u_{0}\right) k
$$

and from Equation (37) we have

$$
B=\left(\partial_{3} u_{2}-\partial_{2} u_{3}\right) i+\left(\partial_{1} u_{3}-\partial_{3} u_{1}\right) j+\left(\partial_{2} u_{1}-\partial_{1} u_{2}\right) k
$$

Therefore, 


$$
\begin{array}{ll}
E_{1}=\partial_{0} u_{1}+\partial_{1} u_{0} & B_{1}=\partial_{3} u_{2}-\partial_{2} u_{3} \\
E_{2}=\partial_{0} u_{2}+\partial_{2} u_{0} & B_{2}=\partial_{1} u_{3}-\partial_{3} u_{1} \\
E_{3}=\partial_{0} u_{3}+\partial_{3} u_{0} & B_{3}=\partial_{2} u_{1}-\partial_{1} u_{2}
\end{array}
$$

Then we find the following identity by carrying out the multiplication,

$$
\begin{aligned}
( & \left.P_{i} \cdot u\right)\left(\bar{P}_{j} \cdot \partial\right)-\left(P_{j} \cdot u\right)\left(\overline{P_{i}} \cdot \partial\right) \\
= & \left(P_{i 0} u_{0}+P_{i 1} u_{1}+P_{i 2} u_{2}+P_{i 3} u_{3}\right)\left(P_{j 0} \partial_{0}-P_{j 1} \partial_{1}-P_{j 2} \partial_{2}-P_{j 3} \partial_{3}\right) \\
& -\left(P_{j 0} u_{0}+P_{j 1} u_{1}+P_{j 2} u_{2}+P_{j 3} u_{3}\right)\left(P_{i 0} \partial_{0}-P_{i 1} \partial_{1}-P_{i 2} \partial_{2}-P_{i 3} \partial_{3}\right) \\
= & \left(P_{i 1} P_{j 0}-P_{j 1} P_{i 0}\right) E_{1}+\left(P_{i 2} P_{j 0}-P_{j 2} P_{i 0}\right) E_{2}+\left(P_{i 3} P_{j 0}-P_{j 3} P_{i 0}\right) E_{3} \\
& +\left(P_{j 2} P_{i 3}-P_{i 2} P_{j 3}\right) B_{1}+\left(P_{j 3} P_{i 1}-P_{i 3} P_{j 1}\right) B_{2}+\left(P_{j 1} P_{i 2}-P_{i 1} P_{j 2}\right) B_{3}
\end{aligned}
$$

For example, with $i=1$ and $j=0$ we obtain the Lorentz transform of $E_{1}$,

$$
\begin{aligned}
\hat{E}_{1}= & \left(P_{11} P_{00}-P_{01} P_{10}\right) E_{1}+\left(P_{12} P_{00}-P_{02} P_{10}\right) E_{2}+\left(P_{13} P_{00}-P_{03} P_{10}\right) E_{3} \\
& +\left(P_{02} P_{13}-P_{12} P_{03}\right) B_{1}+\left(P_{03} P_{11}-P_{13} P_{01}\right) B_{2}+\left(P_{01} P_{12}-P_{11} P_{02}\right) B_{3}
\end{aligned}
$$

and with $i=1$ and $j=3$ we obtain

$$
\begin{aligned}
\hat{B}_{2}= & \left(P_{11} P_{30}-P_{31} P_{10}\right) E_{1}+\left(P_{12} P_{30}-P_{32} P_{10}\right) E_{2}+\left(P_{13} P_{30}-P_{33} P_{10}\right) E_{3} \\
& +\left(P_{32} P_{13}-P_{12} P_{33}\right) B_{1}+\left(P_{33} P_{11}-P_{13} P_{31}\right) B_{2}+\left(P_{31} P_{12}-P_{11} P_{32}\right) B_{3}
\end{aligned}
$$

It is now straightforward to show that the identity in Equation (18) is identical to

$$
\hat{F}=P F P^{t}
$$

where $P^{t}$ is the transpose of the general Lorentz transformation matrix, and $F$ is the electromagnetic field tensor given by Equation (72).

Scientific Research Publishing

\section{Submit or recommend next manuscript to SCIRP and we will provide best} service for you:

Accepting pre-submission inquiries through Email, Facebook, LinkedIn, Twitter, etc. A wide selection of journals (inclusive of 9 subjects, more than 200 journals) Providing 24-hour high-quality service

User-friendly online submission system

Fair and swift peer-review system

Efficient typesetting and proofreading procedure

Display of the result of downloads and visits, as well as the number of cited articles Maximum dissemination of your research work

Submit your manuscript at: http://papersubmission.scirp.org/

Or contact jamp@scirp.org 